\title{
ASCO update 2016
}

\author{
Wolfgang Eisterer $(\mathbb{D}$
}

Received: 13 November 2016 / Accepted: 15 November 2016 / Published online: 7 December 2016

(C) Springer-Verlag Wien 2016

\begin{abstract}
Summary The past few years have seen major progress in cancer research and treatment. Today we are able to select treatments based on molecular profiling of tumors and do not solely rely on tumor type and stage. The most recent advance has taken place in the field of immunotherapy, and at this year's ASCO again a huge number of studies were reported dealing with immunotherapy. In Europe we expect approval of different immunotherapies in various entities ranging from head and neck cancer, urothelial cancer, gastric cancer to lung cancer and colorectal cancer. But immunotherapy is not the only area of progress. There are tremendous advances in surgery, chemotherapy and targeted agents, too, as will be discussed in further detail in this report of the most important abstracts of this year's ASCO by our experts in the field Drs. Wöll, Bartsch, Pall, Roider-Schur, Hamacher, Brodowicz, Kacirek, and myself.
\end{abstract}

Ewald Wöll presents data on curative treatment in locally advanced gastric cancer with negative results for additional postoperative radiochemotherapy as used in the CRITICS study [1]. In the metastatic setting there is no anti-Her2-directed therapy beyond trastuzumab that has shown benefit [2]. Nevertheless, encouraging results were gained with immunotherapy in the Checkmate-032 trial [3] with Nivolumab and the combination of nivolumab and ipilimumab.

Klaus Kacirek discusses the latest findings on the management of colorectal cancer liver metastasis in upfront resectable, borderline resectable, and initially unresectable disease and recommends parenchymal sparing resections for solitary, small metastases in

\section{W. Eisterer $(\bowtie)$}

Klagenfurt Hospital Wörthersee, Klagenfurt, Austria

wolfgang.eisterer@kabeg.at the liver or lung [online first; to be printed in memo 2/2017]. In initially unresectable disease, the focus lies on the induction of high remission rates by triplet chemotherapy plus a biological agent.

Rupert Bartsch weighs the pros and cons in the adjuvant treatment of breast cancer with extended aromatase-inhibition as well as omission of anthracyclines in chemotherapy regimens. In the metastatic setting, a new standard for hormone-receptor-positive patients will appear by the combined action of letrozole and palbociclib, a cyclin-dependent kinase (CDK) $4 / 6$ inhibitor, as demonstrated in the PALOMA-2 trial [4] with impressive improvements in progression-free survival (PFS).

Georg Pall updates us on the most recent findings in lung cancer. There are important findings for a small subset of patients with non-small cell lung cancer (NSCLC) with BRAF-V600E mutations. A combination of targeted agents induced high response rates and long PFS [5]. ALK-rearranged NSCLC seem to benefit more from second-generation ALK inhibition with alectinib than from first-generation with crizotinib as shown in the J-ALEX trial [6].

Sophie Roider-Schur covers data from a FrenchAustrian phase II trial of regorafenib in soft tissue carcinoma (STS) [7] with promising results for this agent in various subtypes of STS. The first results of immune checkpoint inhibitors in STS were presented at this year's ASCO and will be discussed in detail in the manuscript. Exciting data on crenolanib activity in D482V-mutant GIST were presented at the annual meeting and might open the door for a new therapeutic option in an otherwise futile patient segment.

I will summarize data from colorectal cancer. Sidedness of the primary tumor and its predictive role for the use of biological agents in first-line therapy has been one of the most debated topics in this field [8]. 
Different approaches of immune checkpoint inhibition and a combination of this new class of agents have been presented, which I will discuss. Highly exciting data on molecular selection for adjuvant treatment of colorectal cancer were further hot topics.

All in all, scientific and clinical progress is clearly taking place. The possible implications for our daily clinical work will be presented in this issue of memo by our experts. I hope you enjoy the reading and gain new insights for your daily clinical practice.

Yours sincerely, Wolfgang Eisterer

Conflict of interest W. Eisterer declares that there are no competing interests.

\section{References}

1. Verheij M, Jansen EPM, Cats A, et al. A multicenter randomized phase III trial of neo-adjuvant chemotherapy followed by surgery and chemotherapy or by surgery and chemoradiotherapy in resectable gastric cancer: First results from the CRITICS study. J Clin Oncol. 2016;34(suppl):abstract 4000.

2. Kang Y-H, Shah MA, Ohtsu A, et al. A randomized, open label, multicenter, adaptive phase $2 / 3$ study of trastuzumab emtansine (T-DM1) versus a taxane (TAX) in patients (pts) with previously treated HER2-positive locally advanced or metastatic gastric/gastroesophageal junction adenocarcinoma (LA/MGC/GEJC). J Clin Oncol. 2016;34(suppl4S):abstract5.

3. Janjigian YY, Bendell JC, al Calvo Eet. CheckMate032: Phase I/II, open-label study of safety and activity of nivolumab (nivo) alone or with ipilimumab (ipi) in advanced and metastatic (A/M) gastric cancer (GC). J Clin Oncol. 2016;34(suppl):abstract4010.

4. Finn RS, Martin M,HopeS, etal. PALOMA-2: Primary results from a phase III trial of palbociclib (P) with letrozole (L) compared with letrozole alone in postmenopausal women with ER+/HER2- advanced breats cancer (ABC). J Clin Oncol. 2016;34(suppl):abstract524.

5. Planchard D, Besse B, Groen HJM, etal. Anopen-label phase II trial of dabrafenib (D) in combination with rametinib (T) in patients (pts) with previously treated BRAF V600Emutant advanced non-small cell lung cancer (NSCLC; BRF113928). JClin Oncol. 2016;34(suppl):abtract 107.

6. Nokihara H, Hida T, Kondo M, et al. Alectinib (ALC) versus crizotinib (CRZ) in ALK-inhibitor naive ALK-positive nonsmall cell lung cancer (ALK+NSCLC): primary results from the J-ALEX study. J Clin Oncol. 2016;34(suppl):abstract 9008.

7. Mir O, Brodowicz T, Wallet J, et al. Activity of Regorafenib in leiomyosarcomas (LMS) and other types of soft-tissue sarcomas (OTS): results of a double-blind, randomized placebo (PL) controlled phase II trial. J Clin Oncol. 2016;34(suppl):abstract 10504.

8. Vennok AP, Niedzwiecki D, Innocenti F, et al. Impact of primary $\left(1^{\circ}\right)$ tumorlocation on overall survival (OS) and progression-free survival (PFS) in patients (pts) with metastatic colorectal cancer (mCRC): analysis of CAGB/SWOG 80405 (Alliance). JClin Oncol. 2016;34(suppl):abstract3504.

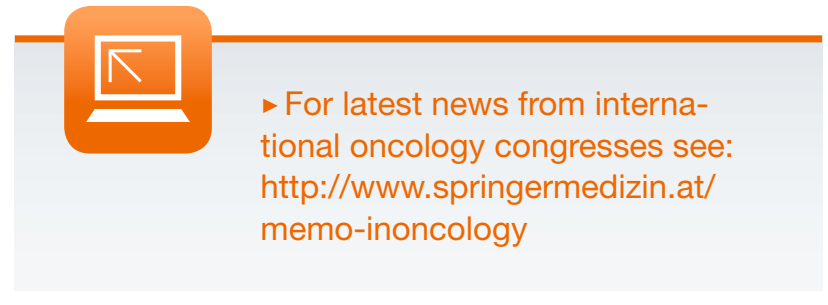

